\title{
The relative crossmodal transfer of a form discrimination '
}

\author{
ROBERT EASTMAN 2 \\ UNIVERSITY OF ARKANSAS
}

In a three-variable factorial design, sets of eight random shapes of high, medium or low geometric complexity were paired with response labels. The stimuli were exposed for 12 sec or 5 sec. During training half the Ss received stimuli in the tactual modality, and the other half got visual stimuli. The groups switched modalities during recognition testing. The T-V sequence produced greater transfer than the $V-T$ sequence.

Studies of the relative crossmodal transfer of form discrimination have yielded discrepant results (e.g., Gaydos, 1956; Ettlinger, 1960; Lobb, 1965). Gaydos (1965) found that visual recognition after tactual training produced significantly fewer errors, in a paired associate task, than tactual after visual. Ettlinger (1960) taught four monkeys a form discrimination, two in the dark (tactually), two under lighted conditions (visually). After training the groups switched conditions for relearning. The results indicated nonsignificant transfer across modalities. In a more recent study (Moffett \& Ettlinger, 1966), monkeys failed to transfer a shape discrimination learned by touch to the visual modality, when compared with controls who did not receive tactual training. These negative results are in contrast with the positive findings of Blakeslee \& Hunter (1966) who also used nonverbal primates (Cebus monkeys) as Ss in a learning set task.

Lobb (1965), using randomly generated angular shapes, had school children identify in one modality a shape previously examined in the other modality. Lobb concluded that the V-T sequence produced greater transfer then its $T-V$ counterpart.

Experimental evidence with humans suggests that there is some common basis for the crossmodal transfer of pattern discrimination. The present study is an attempt to determine the effects of three variables on the crossmodal transfer of a discrimination task: (1) modality sequence, (2) geometric complexity, and (3) the length of stimulus exposure time.

Method

The Ss were 48 University of Arkansas undergraduates. Four Ss were randomly assigned to each of 12 groups in a 2 by 3 by 2 factorial design. The three variables were: (1) visual paired associate training followed by tactual recognition testing $(V-T)$ and tactual training followed by visual testing $(T-V),(2)$ the geometric complexity of the stimuli $(4,8$, and 16 sides), (3) the duration of stimulus exposure (5 and $12 \mathrm{sec}$ ).

Three sets of plane polygonal shapes were used as stimuli. The figures were originally constructed by Vanderplas \& Garvin (1959) according to the first method suggested by Attneave \& Arnoult (1956). They wert, specifically: 4 point-Nos. $2,3,5,9,16,18,25$, 28; 8 point-Nos. $1,3,5,7,17,19,22,26$; and 16 point - Nos. 3, 4, 11, 13, 15, 19, 22, 25 (all of which were randomly chosen from sets of 30 ). The 24 stimuli were constructed so as to be approximately equal in area with variation independent of the levels of complexity.

Visual figures were drawn and filled in with india ink on $3 \times 4$ in. white poster board rectangles. Tactual analogs were cut out of $80 \mathrm{grit}$ sand paper and pasted on $5 \times 5$ in. poster board squares. The visual and tactual versions of the same shape were identical in spatial extent and proportions.

Eight color names were used as identifying responses in the paired associate task. The names were assigned randomly to the stimuli for each $S$ independently.

The testing apparatus consisted of a small table with a shield midway across it which separated $E$ and $S$. The shield had a $3 \times 3$ in. square cut out of it, the bottom edge of which was centered 15 in. above the tabletop. The aperture housed a holder which was used to expose the visual stimuli. A $6 \times 6$ in. square was cut out of the shield for the tactual presentation of stimuli. A black cloth curtain over this opening prevented Ss from seeing $E^{\prime}$ 's side of the table.

A stopwatch was used to time the exposure of stimuli. An eye patch covered S's nonpreferred eye during visual presentation.

During training, Ss were exposed to the tactual (or visual) stimuli for 5 (or 12) sec, and $E$ spoke the color name of a given shape about $5 \mathrm{sec}$ before removing it. Ss were instructed to try to anticipate the response labels on future trials when the same stimuli would be presented in scrambled order. Ss were also told to touch (or view) the next stimulus shape when E signalled by saying "next" (with tactual presentation, however, timing began only when S's preferred hand made contact with the stimuli). For all groups the response label was verbalized by $E$ whether or not $S$ had correctly anticipated it. The interstimulus interval averaged $4 \mathrm{sec}$. The intertrial interval was identical to the interstimulus interval. The interval between the training and test phases averaged $25 \mathrm{sec}$, during which Ss were told to orally emit the color name of any shape recognized during testing. The training criterion was one complete errorless trial (i.e., the correct anticipation of a complete random block of the stimuli).

During testing $\mathrm{E}$ presented the eight stimuli in the "new" modality, and spoke the correct response label 
before each stimulus was removed, whether $S$ had anticipated the response or not. The transfer measure was the number of correct anticipations made by a $S$ during the test trial. Scores could vary from 0 to 8 .

Immediately before training Ss practiced the procedure, under the sequence and time conditions they had been assigned, using three simple dot patterns as stimuli and letters of the alphabet as responses. Resulits

The analysis of variance indicated that the main effect of modality sequence was influential ( $F=13.71$, $\mathrm{df}=1 / 36, \mathrm{p}<.05)$. The means of the $\mathrm{T}-\mathrm{V}$ and $\mathrm{V}-\mathrm{T}$ groups, 3.54 and 5.20 respectively, showed that the $T-V$ sequence produced significantly superior transfer of the shape discrimination than did the $\mathrm{V}-\mathrm{T}$ sequence. Duration of stimulus exposure, geometric complexity, and all interactions were not statistically significant.

The number of trials to criterion during training within sequence groups was not found to be significantly correlated with recognition scores $(r=-0.121, T-V$; $r=0.253, V-T)$, suggesting that differences in transfer scores were not caused by differences in length of training per se.

Discussion

The lack of effect of the complexity variable implies that the complexity levels were not so different psychologically as the geometric dimension would suggest. The number of inflections of a polygon was originally suggested by Attneave (1957) as a dimension which might correlate with psychological complexity. Geometric complexity is related to the rated complexity (visual) of a two dimensional shape (Vanderplas \& Garvin, 1959), but obviously this was not relevant to the present task. Ss may have responded to very lim- ited parts of the stimuli, so that greater complexity, as defined, constituted superfluous information.

The absence of a main effect of duration of stimulus exposure, or a significant sequence by duration interaction suggests that an interval of $5 \mathrm{sec}$ is adequate in either modality for scanning the stimuli used.

The significant main effect of sequence, which indicated the superiority of the $T-V$ order, supports the finding of Gaydos (1956). The superiority of the $T-V$ condition may be accounted for by an observed tendency for $\mathrm{Ss}$ in these groups to compare hand movements with the visual stimuli during testing. The subjective impression of the writer was that the greater difficulty of the training with tactual stimuli also forced the $T-V$ Ss to use verbal identificatory responses to differentiate the stimuli, which facilitated transfer during testing.

\section{References}

ATTNEAVE, F. Physical determinants of the judged complexity of shapes. J. exp. Psychol, 1957, 53, 221-227.

BLAKESLEE, P., \& GUNTER, R. Crossmodal transfer of discrimination learning in Cebus monkeys. Behaviour, 1966, 26 (1-2), 76-90.

ETTLINGER, G. Cross-modal transfer of training in monkeys. Behaviour, $1960,16,56-65$.

GAYDOS, H. F. Intersensory transfer in the discrimination of form. Amer. J. Psychol, 1956, 69, 107-110.

LOBB, H. Vision versus touch in form discrimination, Canad. J. Psychol., $1965,19,175: 187$.

MOFFETT, A., \& ETTLINGER, G. Opposite responding in two sense modalities. Science, 1966, 153 (3732), 205-206.

VANDERPLAS, J. M., \& GARVIN, E. A. The association value of random shapes. J. exp. Psychol., 1959, 57, 147-154.

Notes

1. The results herein formed a part of the author's Master's Thesis. 2. Present address: Department of Psychology, Tulane University, New Orleans, Louisiana. 